\title{
LA UNIVERSIDAD SÉNIOR: \\ DERRIBANDO BARRERAS Y ESTEREOTIPOS EN TIEMPOS DE COVID
}

\author{
Ares-Pernas, Ana Isabel'1; Iglesias-Antelo, Susana²; García Sánchez, María Matilde ${ }^{3}$ \\ ${ }^{1}$ Universidade da Coruña, Departamento de Física y Ciencias de la Tierra, Escuela Universitaria \\ Diseño Industrial \\ ORCID 0000-0003-1183-8733 \\ ${ }^{2}$ Universidade da Coruña, Departamento de Empresa, Facultad de Economía y Empresa, \\ ORCID 0000-0002-4010-4758 \\ ${ }^{3}$ Universidade da Coruña, Departamento de Ciencias de la Salud, Facultad de Enfermería y \\ PodologíaORCID 0000-0001-6712-6351
}

\section{RESUMEN}

En este trabajo se recogen las acciones llevadas a cabo durante el confinamiento del curso 2019/2020 y en el curso 2020/2021 para adaptar la docencia de la Universidad Sénior a la modalidad online. Durante los primeros meses el reto fue tratar de garantizar la continuidad de la actividad formativa, y para ello se diseñó a través de wordpress una herramienta abierta adaptada a las necesidades y circunstancias del alumnado mayor de 50 años. En el curso 2020/2021 se creó el Programa de Formación Específico "Actualidad, Ciencia, Salud y Vida", de carácter semipresencial, en el que se combinaron sesiones online con sesiones presenciales en grupos reducidos. Las sesiones de formación, la atención personalizada y todo el material de apoyo elaborado desde el equipo de la Universidad Sénior, como infografías y videotutoriales, ayudaron al alumnado a alcanzar las competencias digitales que les permitieron seguir la docencia online. De los testimonios recogidos y de la experiencia vivida durante el curso se puede concluir que la pandemia fue una oportunidad para que nuestro alumnado derribase la barrera tecnológica e incorporase las nuevas tecnologías en su vida cotidiana, rompiendo de esta manera muchos de los estereotipos ligados al envejecimiento.

PALABRAS CLAVE: Universidad Sénior, docencia online, competencias digitales, envejecimiento activo, barrera tecnológica. 


\section{CITA RECOMENDADA:}

Ares Pernas, Ana Isabel; Iglesias Antelo, Susana; García Sánchez, Matilde (2021): La Universidad Sénior: Derribando barreras y estereotipos en tiempos de COVID. En García Naya, J.A. (ed.) (2021). Contextos universitarios transformadores: a nova normalidade académica. Leccións aprendidas e retos de futuro. V Xornadas de Innovación Docente. Cufie. Universidade da Coruña. A Coruña (pág. 25 $-40)$

DOI capítulo: https://doi.org/10.17979/spudc.9788497498180.025

DOl libro: https://doi.org/10.17979/spudc.9788497498180

\section{ABSTRACT}

This work describes the main actions carried out during the lockdown period, in the 2019/2020 academic year, and in the 2020/2021 academic year to adapt the Senior University teaching to the online modality. During the first months the challenge was to guarantee the continuity of the learning activity and, for this purpose, an open tool adapted to the needs and circumstances of students over 50 years was designed through wordpress. In the 2020/2021 academic year, the Specific Training Program "Present, Science, Health and Life" was created, with a blended character, in which online sessions were combined with face-to-face sessions in small groups. The training sessions, the personalized attention and all the support material prepared by the Senior University team, such as infographics and videotutorials, helped students to achieve the digital skills that allowed them to follow the teaching online. From our students reflections and from the experience lived during the course, it can be concluded that the pandemic was an opportunity for our students to break down the technological barrier and to incorporate the new technologies in their daily lives, thus breaking many of the stereotypes linked to aging.

KEY WORDS: Senior University, online teaching, digital skills, active aging, technological barrier. 


\section{INTRODUCCIÓN}

El envejecimiento activo es un concepto definido por la Organización Mundial de la Salud como el proceso de optimización de las oportunidades de salud, participación y seguridad con el fin de mejorar la calidad de vida a medida que las personas envejecen (OMS, 2002). Permite a las personas desarrollar su potencial de bienestar físico, social y mental a lo largo de todo su ciclo vital y participar en la sociedad de acuerdo con sus necesidades, deseos y capacidades (Ramos y otros, 2016, Petretto y otros, 2016). Uno de los cuatro pilares del envejecimiento activo es el aprendizaje a lo largo de la vida y es en este sentido donde los programas universitarios para mayores adquieren su principal protagonismo (Ortiz-Colón, 2015, Cambero y Díaz, 2019).

La Universidad Sénior de la Universidade da Coruña (UDC) es un Programa Universitario para Mayores de 50 años que ya no están en activo. Entre sus objetivos destacan difundir el conocimiento y la cultura a través de la formación a lo largo de toda la vida, contribuir a fortalecer la participación colectiva y proporcionar un marco para las relaciones sociales.

Cuando en marzo de 2020 se suspendieron las clases en la Universidad Senior, la actividad tanto académica como social del estudiantado mayor de 50 años fue truncada y una buena parte se vio abocada a una situación de aislamiento y soledad; lo que propició que el equipo de la Universidad Sénior empezara a pensar en nuevas herramientas para poder continuar la actividad formativa de sus estudiantes.

Sin embargo, a pesar de que la tecnología actual puede contribuir a mejorar la vida social y personal de las personas, también es cierto que muchos de nuestros mayores no tienen las competencias digitales necesarias para poder utilizarla (Martínez-Heredia, 2020). Desde la Universidad Sénior nos pareció de vital importancia dar apoyo al estudiantado para adquirir las competencias digitales necesarias para el seguimiento de la docencia online; esto es, proporcionarles la formación y las bases necesarias para este fin (Pascoa y Gil, 2017). Y sin obviar la importancia de romper con muchos de los estereotipos ligados al proceso de envejecimiento, ya 
que los adultos mayores son capaces de manejar las tecnologías de la información y la comunicación con el entrenamiento adecuado (Pino y otros, 2015, López y otros, 2019).

\section{DESCRIPCIÓN DE LA EXPERIENCIA}

Los programas diseñados específicamente para el alumnado que acude a la Universidad Sénior de la UDC no persiguen alcanzar la competencia profesional, sino contribuir a cultivar la mente, propiciar la reflexión sobre la cultura y los valores, y facilitar nuevos conocimientos para aprender a desarrollarse en una sociedad que cambia a ritmo vertiginoso.

Antes de la pandemia, el programa de estudios más tradicional de la Universidad Sénior estaba estructurado en cuatro cursos que se realizaban de forma presencial en sus sedes del Campus de Riazor en A Coruña y del de Esteiro en Ferrol, en los que se impartían materias, seminarios y talleres relacionados con Humanidades y Ciencias Sociales, Ciencia y Tecnología y Ciencias de la Salud, y contaba con un total de 987 estudiantes. En la Figura 1 se puede ver la evolución del número de estudiantes en ambas sedes desde el curso 2004/2005 hasta el curso 2019/2020.

Número de alumnos/as de la Universidad Sénior

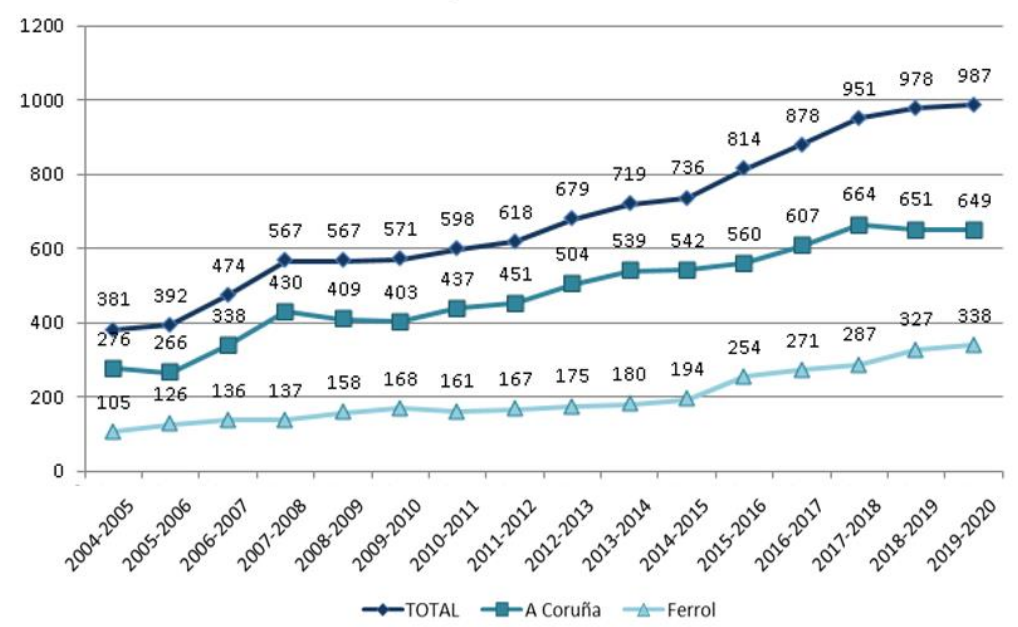

Figura 1. Evolución del número de estudiantes en ambos campus desde el curso 2004/2005 
Cuando en el mes de marzo de 2020 se suspendieron las clases presenciales por la situación de emergencia sanitaria, desde el equipo de la Universidad Sénior quisimos seguir al lado de nuestro alumnado en una situación tan complicada, por lo que se empezó a trabajar para implementar herramientas que ayudasen a garantizar la continuidad de su proceso de enseñanza-aprendizaje durante los días de suspensión de la actividad académica presencial y, sobre todo, que nos permitiesen acompañarlos en esta situación en la que muchos se sentían solos, tal como nos transmitían en sus llamadas telefónicas. El reto era continuar con la actividad formativa de la Universidad Sénior. Para ello se diseñó una herramienta abierta, con el apoyo del personal técnico del Programa, capaz de dar respuesta a las necesidades del alumnado, incluyendo al del programa formativo que se desarrolla en el ámbito rural.

Así, se puso en marcha para todos ellos el espacio web https://universidadesenior.org/ que funcionó a modo de repositorio para los distintos materiales elaborados por el personal docente. En la Figura 2 se presenta una captura de pantalla de la página web donde se puede ver cómo se presentaban los contenidos de las distintas materias y talleres.

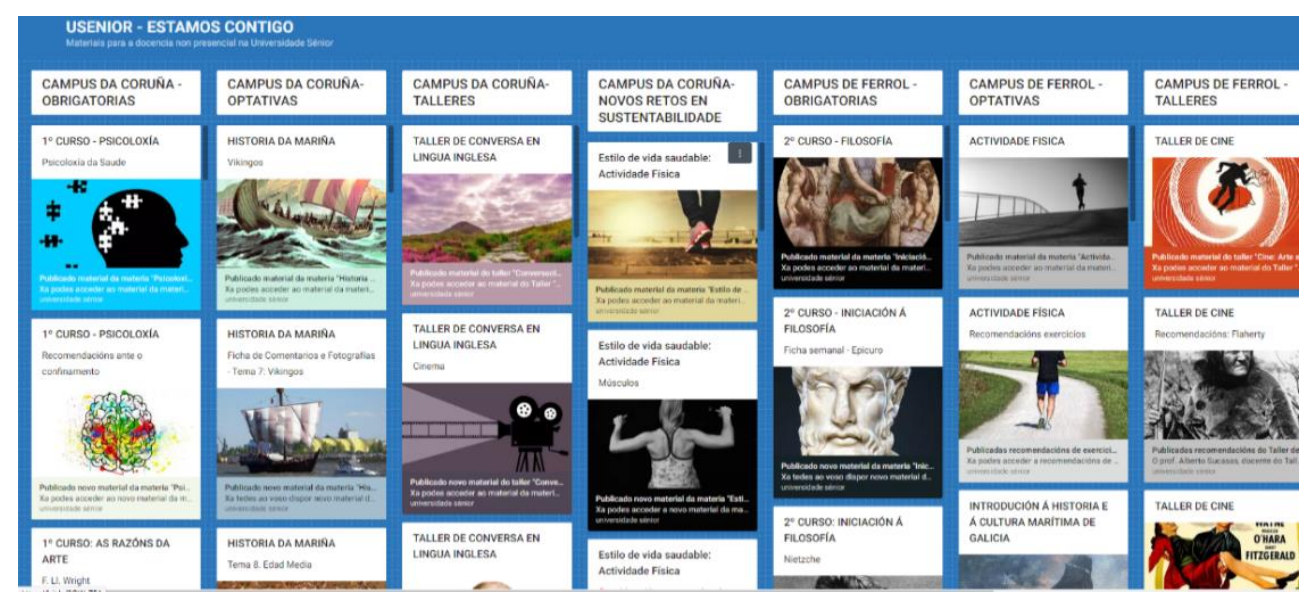

Figura 2. Captura de pantalla del espacio web https://universidadesenior.org/

El alumnado podía acceder también a una serie de recomendaciones a través del espacio USénior estamos contigo, en un formato de revista electrónica semanal. En la Figura 3 se presentan ejemplos de algunos de los consejos compartidos con el alumnado a través de 
ese espacio. Estas actividades online tuvieron un efecto positivo en el aislamiento que en algunos casos sintieron durante el confinamiento.

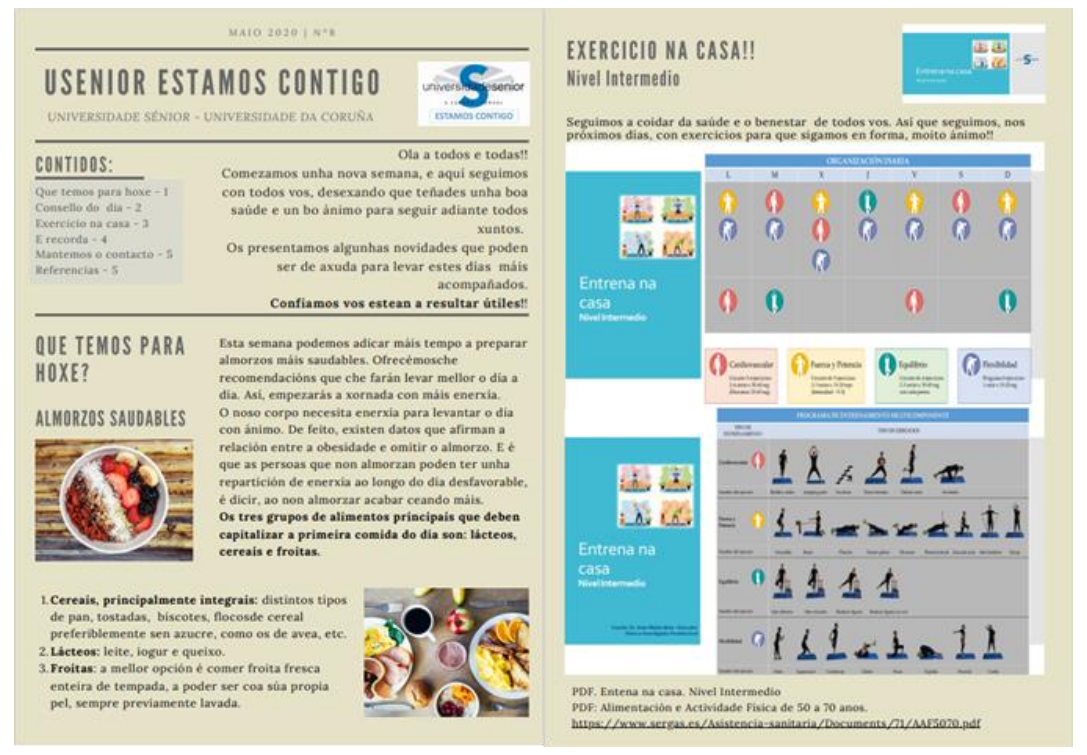

Figura 3. Algunos consejos del espacio USénior estamos contigo

Viendo que la situación se prolongaba, y siendo conscientes de que la presencialidad era un elemento clave para nuestro alumnado, pero sin obviar que, dentro del conjunto de la comunidad universitaria, este es un grupo especialmente sensible, que quizás no tendríamos opción de incorporarnos a la docencia presencial de forma continua a lo largo del curso 2020/2021, y que incluso los programas para mayores de otras universidades se iban a suspender, decidimos conocer la opinión de nuestro alumnado y les hicimos llegar una encuesta en la que les preguntábamos acerca de la posibilidad de continuar en el siguiente curso con formación online. En la encuesta les preguntábamos, además, acerca de las herramientas de las que disponían en sus casas para poder seguir la docencia online.

La respuesta fue muy satisfactoria, ya que un $74 \%$ del alumnado que contestó a la encuesta se manifestó dispuesto a seguir la docencia online, tal y como se puede ver en el gráfico de la izquierda en la Figura 4. Además, se pudo comprobar que la mayoría del alumnado disponía de 
herramientas adecuadas y cómodas para seguir la docencia, como ordenador de sobremesa, portátil 0 tablet (gráfico de la derecha en la Figura 4). También preguntamos a nuestro profesorado acerca de su disposición para impartir docencia online en la Universidad Sénior, a lo que el 95\% respondió afirmativamente (gráfica no mostrada aquí).

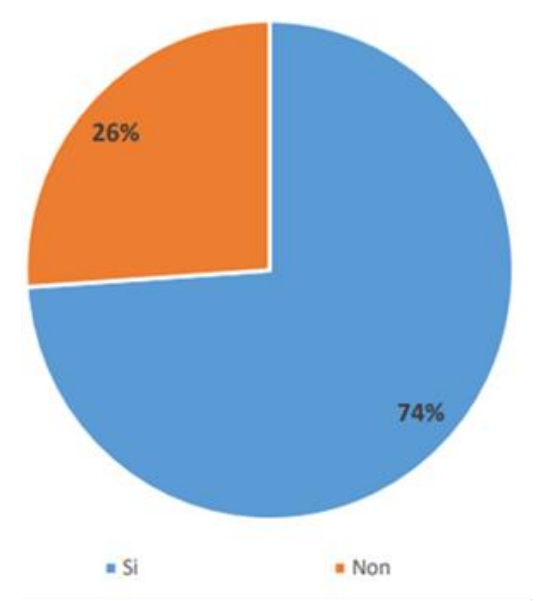

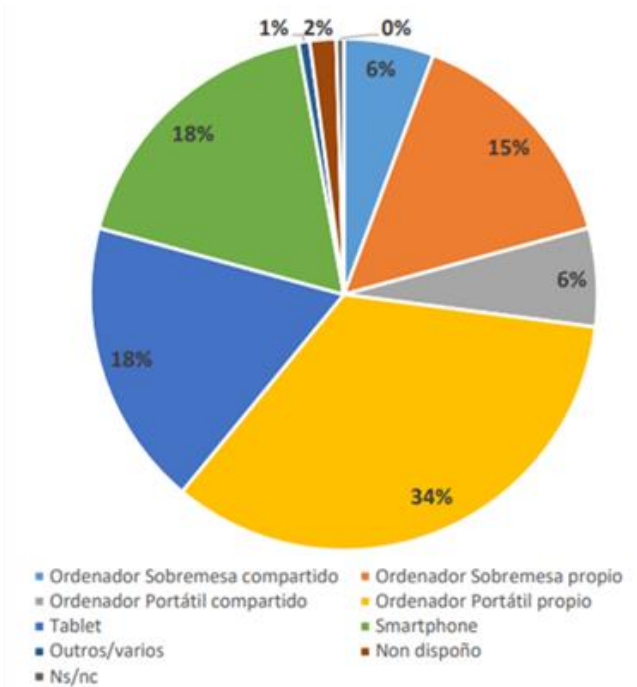

Figura 4. Resultados encuesta docencia online: a) izquierda: ¿estaría dispuesto/a a seguir la docencia online?, b) derecha: ¿de qué dispositivo dispone para seguir la docencia online?

Esta respuesta nos animó a iniciar un proceso de reprogramación de la docencia para el curso 2020/2021, y fue así como nació el Programa de Formación Específico "Actualidad, Ciencia, Salud y Vida", de carácter semipresencial, que se ofreció a todo el alumnado, y en el que finalmente se matricularon casi 400 estudiantes de los dos campus. En el programa se combinaron sesiones de teleformación en remoto a través de las herramientas institucionales de teleenseñanza (Moodle y Teams), de lunes a miércoles, y sesiones presenciales en ambas sedes a través de conferencias o seminarios manteniendo los aforos, los jueves y viernes, con la prioridad absoluta de salvaguardar la seguridad en materia de salud de nuestro alumnado y de la comunidad universitaria en general. En la Tabla 1 se pueden ver las materias cursadas por nuestros estudiantes en modalidad online en ambos campus y algunas de las conferencias realizadas de forma presencial. 


\begin{tabular}{|c|c|c|}
\hline & Campus de A Coruña & Campus de Ferrol \\
\hline 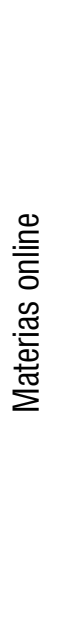 & $\begin{array}{l}\text { Actualidad científica } \\
\text { La economía antes, durante y después de la } \\
\text { pandemia } \\
\text { Los retos de las sociedades contemporáneas } \\
\text { Las ciudades del conocimiento } \\
\text { Las relaciones humanas: una reflexión } \\
\text { filosófica } \\
\text { El estudio de la delincuencia en un mundo } \\
\text { global } \\
\text { Estrategias psicológicas de afrontamiento al } \\
\text { estrés y promoción de la calidad de vida } \\
\text { Educación para la salud. Hábitos saludables } \\
\text { Informática }\end{array}$ & $\begin{array}{l}\text { Estética e ingeniería } \\
\text { Psicología social } \\
\text { La literatura a través de los textos, el arte, el } \\
\text { cine y la música } \\
\text { Actualidad y derecho } \\
\text { Sociedad y naturaleza } \\
\text { Educación para la salud } \\
\text { Introducción a la navegación. Cartas } \\
\text { Náuticas } \\
\text { Clásicos del pensamiento político y su } \\
\text { proyección actual } \\
\text { Informática }\end{array}$ \\
\hline 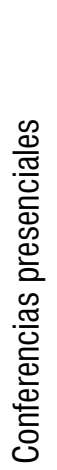 & $\begin{array}{l}\text { Mujeres divinas en las pinturas del Museo } \\
\text { Nacional del Prado } \\
\text { ¿Cómo reducir nuestro impacto ambiental?: } \\
\text { aspectos técnicos y educativos } \\
\text { La neurociencia del sueño: un viaje profundo } \\
\text { La vacunación: una medida preventiva } \\
\text { Conservación y medio marino } \\
\text { El impagable valor de los plásticos en } \\
\text { tiempos del COVID-19 }\end{array}$ & $\begin{array}{l}\text { ¿El amor es ciego? Una reflexión filosófica } \\
\text { sobre la experiencia amorosa } \\
\text { Cuidados ante la artritis reumatoide y la } \\
\text { fibromialgia } \\
\text { Arte e industria: el inicio de un conflicto } \\
\text { Las plantas verdes: } 1000 \text { millones de años } \\
\text { de evolución sobre el planeta } \\
\text { Beneficios de la educación musical a nivel } \\
\text { cognitivo, emocional y terapéutico } \\
\text { Un viaje vital de la lengua gallega }\end{array}$ \\
\hline
\end{tabular}

Tabla 1. Materias y algunas conferencias de la Universidad Sénior en A Coruña y Ferrol

El apoyo del personal técnico y administrativo para pasar al modelo online y capacitar a nuestro alumnado en las nuevas herramientas fue decisivo. Pero no menos importante lo fue la ayuda prestada por nuestros docentes de las materias de Informática. Se programaron sesiones de formación en grupos reducidos en los meses de julio y septiembre en las que se capacitó al alumnado para consultar el correo electrónico, conectarse a través de Teams o descargar materiales en el Campus Virtual. También se les proporcionaron infografías y tutoriales preparados desde el equipo de la Universidad Sénior (Figura 5). Además, el alumnado que presentó más dificultades contó con tutorías individuales y personalizadas durante todo el curso. 
En contra de lo que pudiese parecer, a finales de octubre nuestro alumnado era capaz de seguir la docencia online, participar en los debates planteados por el personal docente a través de Teams y desenvolverse con soltura en el Campus Virtual. En definitiva, había alcanzado las competencias digitales requeridas para la docencia no presencial.
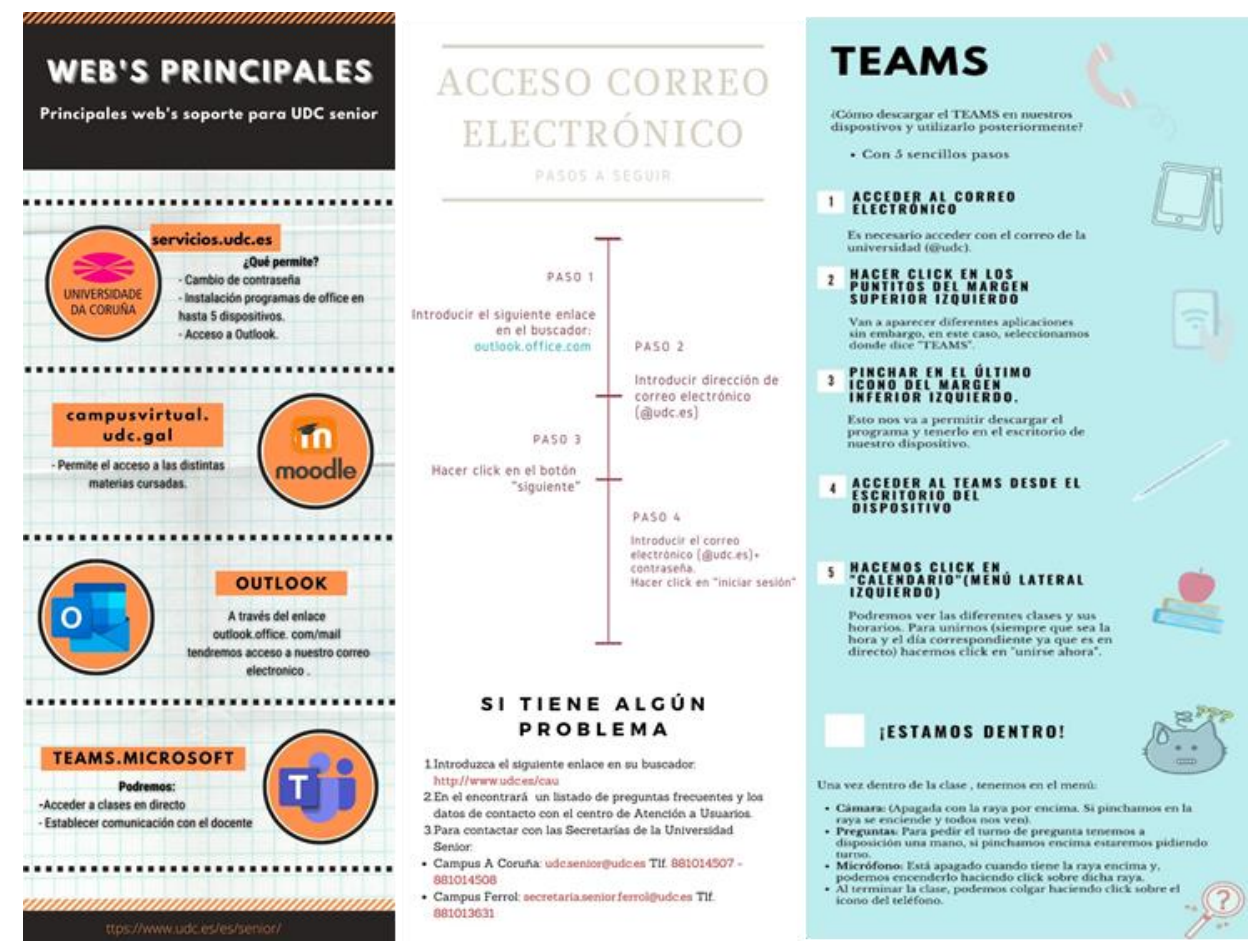

Figura 5. Infografías diseñadas como apoyo al alumnado

\section{RESULTADOS}

Para evaluar el resultado de la experiencia semipresencial, en el mes de abril, con el primer cuatrimestre finalizado y el segundo bastante avanzado, nos pareció de gran interés reunirnos con nuestro alumnado y recoger algunas de sus reflexiones, que presentaremos a continuación. En la Figura 6 se pueden ver imágenes de algunas de esas entrevistas. También, se recogieron encuestas de satisfacción tanto con la modalidad online de la docencia, como con la docencia impartida por cada miembro del profesorado. 


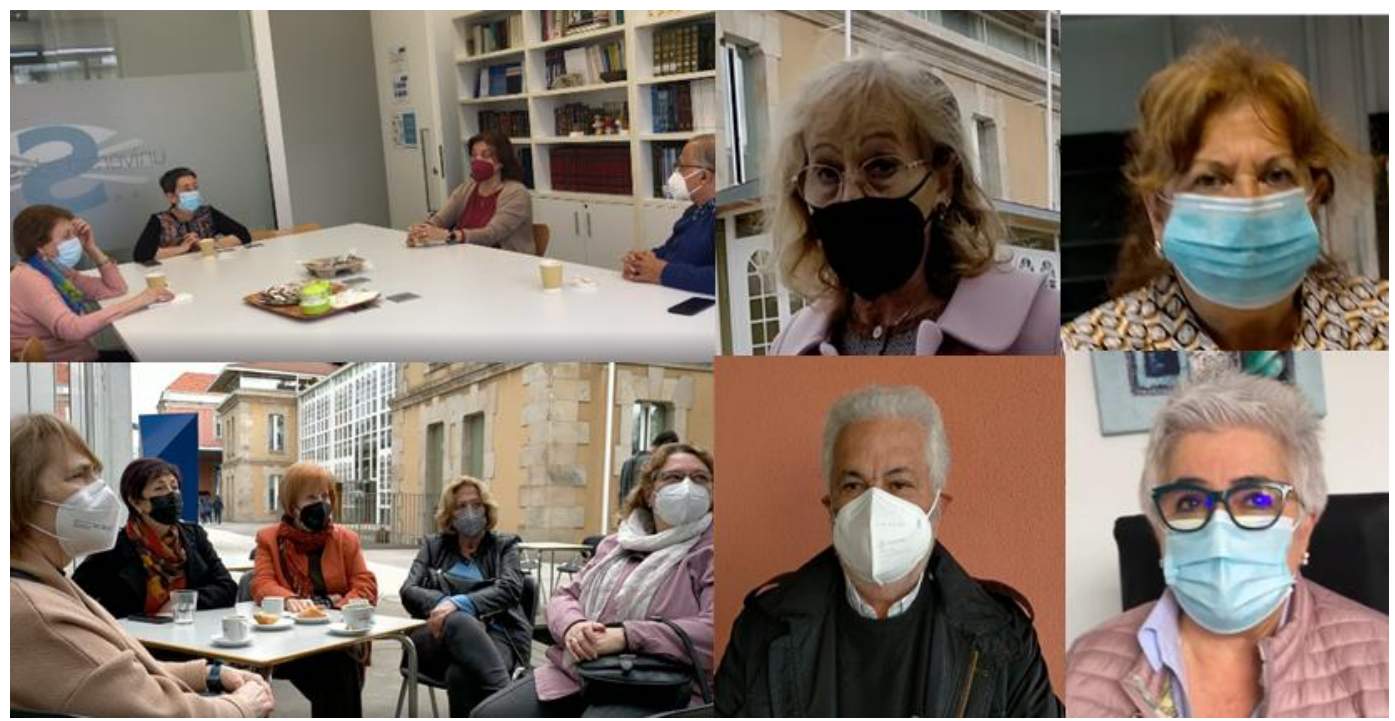

Figura 6. Imágenes de algunas de las entrevistas con nuestro alumnado

Del análisis de las respuestas de nuestro estudiantado pudimos constatar en primer lugar, lo acompañado y valorado que se sintió por la Universidad de A Coruña, en general y por la Universidad Sénior, en particular:

"Fue una sorpresa que la Universidad Senior nos tuviera en cuenta para la docencia... se nos ha tratado como universitarios, como parte de la comunidad..." (mujer, USénior Coruña).

"La docencia online me pareció una cosa muy buena para no cortar la enseñanza en la universidad" (hombre, USénior Ferrol).

"Nos han dado un valor, no nos dejaron arrinconados, nos abrieron una ventana donde pudimos entrar" (mujer, USénior Coruña).

También se pudo vislumbrar que, a pesar de los miedos iniciales, unirse a la docencia online supuso para ellos un nuevo reto que estaban dispuestos a asumir y una nueva ilusión y un modo de sentirse acompañados en una época tan complicada: 
"Empecé a animar a todo el mundo alrededor, a decirles que era lo mismo, que podíamos hablar, que podíamos escribir que podíamos participar en clase, lo único que no podíamos era tocarnos" (mujer, USénior Coruña).

"Si los demás estudiantes tenían actividad virtual también nosotros podemos, estamos capacitados para eso y para mucho más..." (hombre, USénior Coruña).

“De mantener una vinculación con la Sénior que nos había dado tanto... en una situación como la que estuvimos viviendo de cuarentena, de estar encerradas, para nosotros era una ventanita el ordenador de estar en contacto con compañeros, con la cultura, obvio, y seguir nuestra vida, una disciplina..." (mujer, USénior Ferrol).

"Después de la soledad del encierro que a mí me dejó psicológicamente mal, que me ofrecieran tener la clase... no me paré ni a pensarlo... cada día que tengo clase es como un objetivo... es como si volviese a tener mi vida organizada" (mujer USénior Coruña).

"Mucha gente está sola en casa, por tanto, cuando abro el ordenador tengo esa sensación de formar parte de algo, de estar en algún sitio, de tener ese vínculo... por eso psicológicamente y culturalmente fue un favor a toda la comunidad” (mujer, USénior Coruña).

Aunque obviamente reconocen haber tenido problemas informáticos en los momentos iniciales, en general, afirman haberse adaptado bien gracias al apoyo del personal técnico y administrativo de la Universidad Sénior y de los profesores de la materia de Informática. También valoran muy positivamente el hecho de haber aprendido a manejar el Campus Virtual (Moodle) o los archivos de Teams para poder consultar los materiales de las materias, y el haber tenido la oportunidad de romper la brecha digital. Reconocen el valor de las familias como apoyo en el proceso de adaptación al modelo online: 
"A pesar de haber tenido problemas informáticos ha sido una experiencia muy positiva" (mujer, USénior Coruña).

"Tuvimos una gran ayuda con la clase de informática, eso me resolvió algunas dudas iniciales" (hombre, USénior Ferrol).

"Después vas a Moodle y completas los apuntes" (mujer, USénior Coruña).

"Es fantástico que cuando acabas la clase puedas ver la grabación otra vez" (mujer, USénior Coruña).

"Yo no quería quedar atrasada y analfabeta digital" (mujer, USénior Ferrol).

"Empezamos a usar el ordenador de otra manera... a mí me dio un poco de miedo meterme en este mundo, pero las clases de informática, el personal de administración, los profesores, la dirección e incluso nuestras familias, todos, nos ayudaron mucho" (mujer, USénior Ferrol).

Valoran de forma muy positiva la oportunidad de asistir al menos una vez al mes a una conferencia presencial:

"Yo me apunté online con la esperanza de que volviéramos a presencial. Al final no pudo ser, pero estuve encantada con las conferencias presenciales de los jueves" (mujer, USénior Ferrol).

"Sabiendo que con los tiempos tan malos que fueron tuve la oportunidad de ir a unas conferencias de vez en cuando... me siento privilegiada" (mujer, USénior Ferrol).

En general, todos evalúan muy positivamente la experiencia online como modo de continuar la docencia en esta situación excepcional e incluso reconocen que algunos que no se animaron a matricularse en el programa semipresencial se arrepintieron de no haberlo hecho: 
"Mi experiencia con la docencia online ha sido buena y animaría a todo el mundo a que participase" (hombre, USénior Ferrol).

"La satisfacción con el programa ha sido muy buena porque los profesores y la dirección han hecho un programa muy bien adaptado a esta situación de la COVID19" (hombre, USénior, Ferrol).

"Los que no se apuntaron ahora están deseosos de poderse apuntar" (hombre, USénior Coruña).

En cuanto a los resultados de las encuestas, todos mostraron de igual modo su satisfacción con la docencia online y con el profesorado en general, y sólo en algunos de los casos reconocieron tener algunas dificultades como se puede ver en la Figura 7. Entre ellas destacan el aprender a manejar las herramientas y el hecho de interactuar durante la clase (encender micrófonos, cámaras, levantar la mano e incluso respetar el turno de palabra).

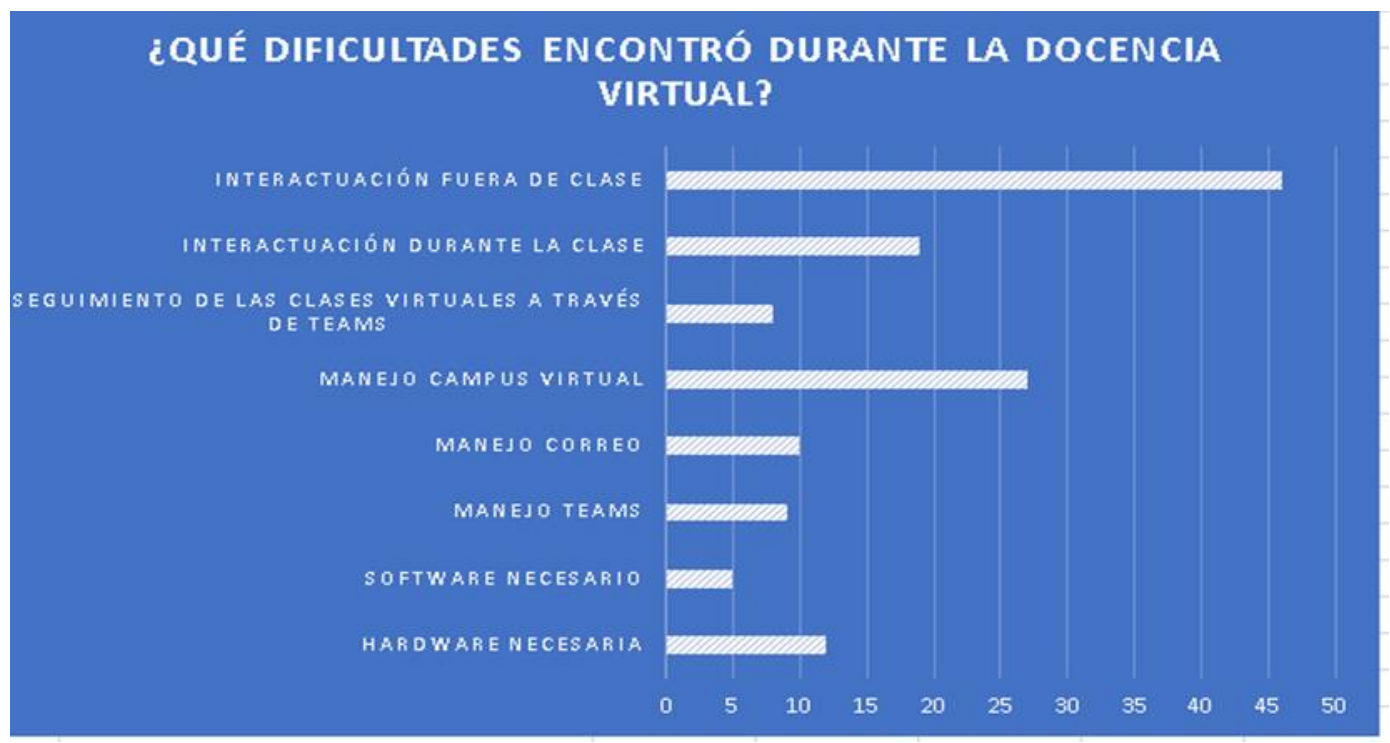

Figura 7. Dificultades encontradas por el alumnado en la docencia virtual 


\section{CONCLUSIONES}

Es importante destacar que en la actualidad tenemos un nuevo perfil de personas mayores que se desmarca de los estereotipos sociales característicos de las últimas décadas. Las personas mayores tienen mucho que aportar al mundo, son proactivas, disfrutan del momento e intentan integrar en sus vidas las nuevas tecnologías lo que les hace estar actualizadas, informadas y en constante comunicación con su entorno; por lo que, en contra de lo que pudiese parecer, su transición al formato online no resultó tan complicada.

Para el equipo de la Universidad Sénior fueron meses de trabajo intenso. Era necesario realizar una reprogramación de la docencia, adaptando herramientas y espacios. Este logro se alcanzó gracias a un trabajo multidisciplinar en el que cada miembro aportó su conocimiento, generando así unas sinergias muy positivas. Afortunadamente, pudimos contar con todo el apoyo del Centro Universitario de Formación e Innovación Educativa (CUFIE) y de los servicios informáticos y de gestión de la UDC, que nos apoyaron en la creación de grupos de docencia, en la adaptación de las plataformas a nuestras necesidades, y en la solución de problemas técnicos con las mismas. $Y$ también el profesorado tuvo que asumir un nuevo reto: la transformación de la docencia a modalidad online dirigida a un público exigente como lo es el alumnado de la Universidad Sénior.

A pesar de todo, la satisfacción de nuestro estudiantado con la nueva docencia online y por el hecho de sentirse acompañado y activo durante la pandemia es razón suficiente para creer que el esfuerzo ha merecido la pena. Además, consideramos que la pandemia fue una oportunidad para que los mayores pudiesen dar el salto tecnológico e incorporar las nuevas tecnologías en su vida cotidiana, derribando de esta forma muchos de los estereotipos ligados al envejecimiento.

Aunque en el próximo curso se pretende retomar la actividad presencial, nos parece importante mantener el programa online para el alumnado que decida continuar en esta 
modalidad. En este sentido, el uso de herramientas de teleenseñanza para la docencia y la competencia digital del alumnado desempeñarán un papel importante en el proceso de enseñanza-aprendizaje del Programa para Mayores de la Universidad Sénior.

\section{REFERENCIAS}

Cambero, S. C. \& Díaz, D. (2019). Aprendizaje a lo largo de la vida como estrategia de envejecimiento activo. Caso de estudio de la Universidad de Mayores de Extremadura. Revista de Sociología de la Educación, 12 (1), pp. 104-122.

López, F., de San Pedro, M., Aumatell, E., Simó, S. \& García, F. (2019). An intergenerational information and communications technology learning project to improve digital skills: user satisfaction evaluation. JMIR Aging, 2 (2), doi: 10.2196/13939.

Martínez-Heredia, N. (2020). Current challenge in the digital age: ICT and the elderly at University of Granada (Spain). Texto Livre, 13 (1), pp. 82-95.

Organización Mundial de la Salud (2002). Envejecimiento activo: un marco políico. Revista Española de Geriatría y Gerontología, 37 (2), pp. 74-105.

Ortiz-Colón, A. M. (2015). Los programas universitarios de personas mayores y el envejecimiento activo. Formación Universitaria, 8 (4), pp. 55-62.

Pascoa, G. \& Gil, H. (2017). The urgency of lifelong learning: A study on the learning of information and communication technologies in populations 50+. 12th Iberian Conference on Information Systems and technologies (CISTI), doi: 10.23919/CISTI.2017.7976002.

Petretto, D. R., Pili, R., Gaviano, L., Matos, C. \& Zuddas, C. (2016). Envejecimiento activo y de éxito o saludable: una breve historia de modelos conceptuales. Revista Española de Geriatría y Gerontología, 51 (4), pp. 229-241.

Pino, M. R., Soto, J. G. \& Rodríguez, B. (2015). Las personas mayores y las TICs. Un compromiso para reducir la brecha digital. Pedagogía social. Revista Interuniversitaria, 26, pp. 337-359. 
Ramos, A. M., Yordi, M. \& Miranda, M. A. (2016). El envejecimiento activo: importancia de su promoción para sociedades envejecidas. Revista Archivo Médico de Camagüey, 20 (3), pp. 330-337. 